United Nations Educational Scientific and Cultural Organization

and

International Atomic Energy Agency

THE ABDUS SALAM INTERNATIONAL CENTRE FOR THEORETICAL PHYSICS

\title{
WORK FUNCTIONS AND SURFACE CHARGES AT METALLIC FACET EDGES
}

\author{
C.J. Fall \\ Institut Romand de Recherche Numérique en Physique des Matériaux \\ and Institut de Théories des Systèmes Physiques, \\ École Polytechnique Fédérale de Lausanne, CH-1015 Lausanne, Switzerland, \\ N. Binggeli \\ The Abdus Salam International Centre for Theoretical Physics, Trieste, Italy \\ and \\ A. Baldereschi \\ Institut Romand de Recherche Numérique en Physique des Matériaux \\ and Institut de Théories des Systèmes Physiques, \\ École Polytechnique Fédérale de Lausanne, CH-1015 Lausanne, Switzerland \\ and \\ INFM-Dipartimento di Fisica Teorica, Universitá di Trieste, I-34014 Trieste, Italy.
}

\begin{abstract}
The electronic charge densities and work functions at sharp metallic facet edges are determined from $a b$ initio calculations, combined with macroscopic averaging techniques. In particular, we examine how two different work functions coexist at close range near edges between inequivalent facets. The surface ionic relaxation at facet edges is shown to influence appreciably the local electrostatic potential in the vacuum. Various edges between $\mathrm{Al}(100)$ and $\mathrm{Al}(111)$ facets are studied, as well as between $\mathrm{Na}(110)$ facets. We also develop a model of electronic surface dipoles, which accounts for the surface charge transfer between inequivalent facets, and which allows us to predict the influence of the shape and size of a macroscopic crystal on its work functions.
\end{abstract}

MIRAMARE - TRIESTE

April 2002 


\section{INTRODUCTION}

Microscopic details in the electronic density at metal surfaces are responsible for the dependence of the work function on the crystal surface orientation. ${ }^{1}$ This dependence, i.e., the work function anisotropy, is a quantum phenomenon that has clear macroscopic consequences, since differences in work function of up to $1 \mathrm{eV}$ can be measured between two surfaces of a given element. ${ }^{2}$ This leads to an interesting problem, namely the spatial dependence of the work function outside a finite crystal, particularly if non-equivalent facets are exposed to the vacuum. At large distances from a finite crystal, the crystalline anisotropy is negligible, and the electrostatic potential and work function must be isotropic and constant. Closer to the crystal, the electrostatic potential must vary instead from facet to facet, mirroring the changes of the surface work functions. Since the Fermi energy is uniform within a crystal and the electrostatic potential outside a smooth surface is constant beyond about $1 \mathrm{~nm}$, the changes in the vacuum potential must be eminently local at facet edges. Several experimental techniques allow nowadays work functions of different crystal facets to be measured simultaneously. For example, using a single-crystal hemispherical cathode, the work functions of several surface orientations have been imaged simultaneously by thermionic projection. ${ }^{3}$ The local work function near facet edges has also been mapped by scanning probe-hole field-emission microscopy. ${ }^{4}$ However, the mechanism allowing for different surface work functions to exist simultaneously near a crystal edge has remained largely unexplored up to now. Also, the effects of facet edges on the local work function are not well understood yet.

In a previous letter, ${ }^{5}$ we examined, in a particular case, the problem of different work-function coexistence near a facet edge. The apparent work function-defined as the difference between the vacuum potential at an infinite distance from a crystal and the Fermi level — was also shown to depend in general on the global crystal morphology. ${ }^{5}$ The purpose of this article is to present, in more detail, and by means of further $a b$ initio calculations, the behavior of the electronic charge and the potentials they induce close to a variety of facet edges. We also investigate the effects of surface ionic relaxation and of image potential contributions to the local work function.

We concentrate here on atomically-sharp edges of high-density low-index facets. Previous theoretical investigations of the electrostatic potential around sharp metallic edges have been limited to studies of a $90^{\circ}$ jellium wedge. An early description concentrated on the potential inside the metal, ${ }^{6}$ modeling the electronic density by a product of two one-dimensional surface distributions. The $90^{\circ}$ wedge has also been studied more recently in the Thomas-Fermi approximation in relation with edge energies. ${ }^{7}$ However, this model is known to be a poor approximation for surfaces, since the associated work function vanishes for all electronic densities of the bulk metal. ${ }^{8}$ Experimentally, atomic-sharp edges are accessible by build-up of low-index facets due to surface diffusion in the presence of an electric field, for example. This technique has been exploited in microtip fabrication for scanning tunneling microscopy. ${ }^{9}$ 
As prototype systems, we focus on aluminum, an $s p$ metal, and on sodium, a simple $s$ electron metal, for comparison. The $\mathrm{Al}$ work function has been studied theoretically by several authors, notably using discrete-lattice corrections to a jellium surface, ${ }^{10,11}$ semi-analytic variational calculations, ${ }^{12}$ density functional theory, ${ }^{13}$ and a one-dimensional self-consistent model with Ashcroft pseudopotentials. ${ }^{14}$ These calculations all describe infinitely-extended single surfaces and obtain some anisotropy in the metal work function. The Al work function increases experimentally from (111) to (110) and to (100). ${ }^{15}$ This trend is different from that observed for most fcc metals, which show increasing work functions with increasing surface atomic packing, from (110) to (100) and to (111). In a previous study, ${ }^{16}$ we have shown that the observed trend in $\mathrm{Al}$ can be explained by the $p$-atomic-like character of the density of states at the Fermi

energy. Conversely, sodium, a bcc metal, displays a work function anisotropy ${ }^{16}$ that follows Smoluchowski's rule of lower work functions for more open surfaces. ${ }^{17}$

After briefly introducing the theoretical methodology of our calculations in Section II, we will determine the charge density, in Section III, and the local work function, in Section IV, near an edge between two equivalent $\mathrm{Al}$ and $\mathrm{Na}$ facets, as well as between inequivalent $\mathrm{Al}$ facets. The importance of ionic surface-relaxation effects near facet edges will be highlighted in Section V. The long-range features of the electrostatic potential outside a finite metal crystal will be understood in terms of a simple model of the surface dipole in Section VI, before concluding in Section VII.

\section{THEORETICAL METHODOLOGY}

We perform $a b$ initio calculations within density functional theory (DFT) in the local density approximation (LDA) using the Ceperley-Alder exchange-correlation functional. ${ }^{18} \mathrm{We}$ use Troullier-Martins pseudopotentials ${ }^{19}$ in the Kleinman-Bylander approximation. ${ }^{20} \mathrm{~A}$ set of Monkhorst-Pack special k-points are employed for the Brillouin zone integrations, ${ }^{21}$ together with a Gaussian broadening of 0.01 Ry to determine the position of the Fermi level. ${ }^{22}$ The valence charge density is determined self-consistently by expanding the electronic wave functions on a set of plane waves with kinetic energies up to $16 \mathrm{Ry}$. Ionic relaxations near facet edges are optionally included by allowing the outermost layer of ions to move to their equilibrium positions.

We concentrate in this study on $\mathrm{Al}(100)$ and $\mathrm{Al}(111)$ facets and their mutual edges, and on $\mathrm{Na}(110)$ facets for comparison. The surface potentials near facet edges are determined by considering a supercell describing a nanowire of polygonal section with flat facets of low-index orientations. The crystal retains the bulk periodicity in one dimension, and thus describes infinitely-long nanowires and facet edges. The lateral periodic boundary conditions on the supercell introduce parallel replicas of the wire, which are separated by vacuum regions. The supercell is chosen with basis vectors parallel to the crystal facets. For the facet edge between 
$\mathrm{Al}(100)$ and $\mathrm{Al}(010)$, respectively $\mathrm{Al}(111)-\mathrm{Al}(11 \overline{1})$, we used a $5 \times 5$ array of atoms, with a distance equivalent to 5 vacuum planes along each dimension and 7 , respectively 10 , reduced k-points. For the $\mathrm{Al}(100)-\mathrm{Al}(111)$ edge, we used a $5 \times 5$ array of atoms, with 4 vacuum planes parallel to the (111) facet and 5 vacuum planes parallel to the (100) facet, with 8 k-points. For the sodium nanowire, we use a $6 \times 6$ array of atoms to model $\mathrm{Na}(110)$ facets, with 6 equivalent vacuum layers in each direction, and 8 reduced k-points.

We evaluate the accuracy of our procedure by comparing the nanowire surface potentials with infinitely-extended surface calculations. For such surfaces, studied with a standard thinfilm supercell approach in conjunction with macroscopic averages, the computed work functions are 4.25, 4.38, and $4.30 \mathrm{eV}$ for the $\mathrm{Al}(111), \mathrm{Al}(100)$ and $\mathrm{Al}(110)$ surfaces respectively, ${ }^{23}$ compared to $4.24,4.41$, and $4.28 \mathrm{eV}$ experimentally. ${ }^{15}$ The theoretical values agree to within $0.03 \mathrm{eV}$ with experiment and successfully reproduce the experimental trend. The uncertainty on the absolute value of the work function due to the LDA is typically of the order of $0.1 \mathrm{eV}$. However, as usual within the LDA, and consistent with gradient-correction calculations (GGA), we expect a better accuracy for the relative values of the work functions, on the order of the numerical convergence of our results with the computational parameters, i.e., $\sim 0.03 \mathrm{eV}$. For comparison, when using the GGA with the Perdew-Burke-Ernzerhof functional, ${ }^{24}$ the work functions of the $\mathrm{Al}(100)$ and $\mathrm{Al}(111)$ surfaces shift downward by $0.17 \mathrm{eV}$, compared to the LDA results. The local work functions around nanowires are also reduced by about the same amount $(0.15-0.17 \mathrm{eV})$.

\section{CHARGE DENSITIES NEAR FACET EDGES}

To evaluate the surface dipole near facet edges, we use a two-dimensional (2D) macroscopic averaging procedure. Once the three-dimensional charge density $\rho\left(x_{1}, x_{2}, x_{3}\right)$ is determined $a b$ initio, a linear average parallel to the facets is performed to obtain a $2 \mathrm{D}$ average charge density:

$$
\bar{\rho}\left(x_{1}, x_{2}\right)=\frac{1}{l_{3}} \int_{0}^{l_{3}} d x_{3} \rho\left(x_{1}, x_{2}, x_{3}\right),
$$

where the coordinates $x_{1}, x_{2}, x_{3}$ are measured along the supercell edges with the third axis chosen along the nanowire axis. $l_{3}$ is the thickness of the supercell parallel to the facet edges. The microscopic crystal periodicity is evacuated to magnify surface and edge effects on the charge density and potential by evaluating a 2D macroscopic average:

$$
\overline{\bar{\rho}}\left(x_{1}, x_{2}\right)=\frac{1}{A} \int_{-l_{1} / 2}^{l_{1} / 2} d x_{1}^{\prime} \int_{-l_{2} / 2}^{l_{2} / 2} d x_{2}^{\prime} \bar{\rho}\left(x_{1}+x_{1}^{\prime}, x_{2}+x_{2}^{\prime}\right),
$$

where $l_{1}$ and $l_{2}$ are the dimensions of the $x_{3}$-projected microscopic lattice unit cell, and $A$ is its area.

This averaging technique is illustrated in Fig. 1 in the case of a facet edge between two equivalent $\mathrm{Al}(100)$ surfaces. The self-consistent valence electron charge density projected parallel to the facet edge and the macroscopic average of the total charge (valence-electron plus ion-point 
charge) are shown, thereby highlighting the surface dipole. The electronic density smooths the rectangular area of the macroscopic ionic charge at the facet edge. The influence of the facet edge on the charge density extends essentially up to the atomic columns surrounding the edge, beyond which densities characteristic of infinite crystal faces are recovered.

We now turn to the charge density at a facet edge between two inequivalent surfaces. Fig. 2 shows the total macroscopic charge around the two different edges between $\mathrm{Al}(100)$ and $\mathrm{Al}(111)$ facets, without any surface relaxation. Near the obtuse edge, the electronic density closely follows the angled surface, with slight smoothing within the bulk part (within $\sim 2$ a.u.). Around the acute angle, the smoothing is notably stronger. The intrinsic difference between the two facets is not readily seen in the contour plot of the charge density, but leads, as we will see in the next section, to noticeable differences in the electrostatic potential.

\section{LOCAL WORK FUNCTION AROUND FACET EDGES}

Outside a crystal surface, beyond the extent of the electronic charge density, the local work function is governed by both the image potential and the difference between the local electrostatic potential and the Fermi energy of the crystal. It is well known that the LDA incorrectly describes the asymptotic form of the image potential. The LDA exchange-correlation potential falls off exponentially, whereas the actual non-electrostatic potential contribution to the local work function should follow the classical image potential beyond a few atomic units from the surfaces. ${ }^{25}$ The latter contribution, however, known analytically for metallic edges of arbitrary angles, plays no role in the coexistence of different surface work functions near facet edges, nor in the apparent work function at an infinite distance from the crystal. In the Appendix, we explicitly determine the behavior of the classical image potential around two prototype metal wedges (a $90^{\circ}$ and an acute-angle wedge). Because the image potential becomes negligible at sufficient distance $(\sim 100 \mathrm{~nm})$ from any crystal-sample surface, the apparent work function is entirely determined by the electrostatic potential contribution. The same applies to the workfunction values of the infinitely-extended surfaces, and hence to the coexistence mechanism. Moreover, as the image potential behaves monotonously around facet edges (see appendix) any local variation in the work function around facet edges should also derive from the electrostatic potential contribution. In what follows, we will therefore focus on the electrostatic component, $W_{E}$, of the local work function.

To calculate the electrostatic potential at large distances from the nanowire, we must use a technique to remove its supercell neighbors. The macroscopic electrostatic potential $\overline{\bar{v}}\left(x_{1}, x_{2}\right)$ outside an isolated wire is determined from the corresponding macroscopic average of the total charge $\overline{\bar{\rho}}\left(x_{1}, x_{2}\right)$ by means of a modified 2D multipole scheme. ${ }^{26}$ In this technique, the plane is

divided into two regions by a circle surrounding the total macroscopic charge of a single nanowire (within its supercell). Inside this ring, we determine the electrostatic potential from the self- 
consistent charge density by solving the Poisson equation in reciprocal space, using the supercell periodic boundary conditions. The potential is also determined in this way at discrete points uniformly spread around the circle. Considering then that the ring is in a vacuum, without any supercell neighbors, the potential outside the ring can be expanded in terms of this set of values. ${ }^{26}$ The isotropic potential at infinity, which corresponds to the apparent work function, can be computed from the average of the potential values calculated around the ring. The local work function $W_{E}\left(x_{1}, x_{2}\right)$ is obtained by subtracting the Fermi level $E_{F}$ from the macroscopic electrostatic potential $\overline{\bar{v}}\left(x_{1}, x_{2}\right)$ :

$$
W_{E}\left(x_{1}, x_{2}\right)=\overline{\bar{v}}\left(x_{1}, x_{2}\right)-E_{F} .
$$

The Fermi energy $E_{F}$ is found from a separate bulk calculation ${ }^{27}$ to reduce quantum size effects. ${ }^{28}$

The electrostatic potential created around a wire formed from $\mathrm{Al}(100)$ facets, without any surface relaxation, is shown in Fig. 3. The bulk Fermi energy has been subtracted, thus obtaining a representation of the local work function $W_{E}$. We note that a macroscopically flat potential, indicating a bulk behavior of the charge density, is obtained within an extended section of the wire. Outside the center of each facet, we obtain a local work function close to the infiniteplane work function. Toward the edges, the potential dips markedly and the contour lines extend further into the vacuum. A similar picture was obtained in the theoretical study of stepped jellium surfaces, ${ }^{29}$ where the potential contour lines were seen to protrude near the top of the ledges, on account of the reduced electronic charge density in those regions. Scanning tunneling microscopy images of the local work function of $\mathrm{Au} / \mathrm{Cu}(111)$ surfaces have also shown a lowering of the potential around step edges. ${ }^{30}$ However, the aspect of the electrostatic potential calculated here $a b$ initio at a facet edge is strikingly different from that obtained for a jellium in the Thomas-Fermi approximation, ${ }^{7}$ where only a near-monotonous smoothing around the edge was observed. This difference highlights the importance of self-consistent field approaches when studying facet edges.

When leaving this nanowire in a [110] direction, along a diagonal of the square nanowire section, the potential increases all the way to its value at infinity. Perpendicularly to the $\mathrm{Al}(100)$ facets, the potential instead shows a broad maximum corresponding to the value of the infinite-plane work function, before decreasing to its value at infinity. The latter variation indicates that the edge influences the potential value at infinity. This feature is related to the nanoscopic lateral size of our wire. Indeed, the characteristic length $\left(d_{m}\right)$ associated with the electron smoothing at edges is of the order of a few angstroms. ${ }^{5}$ In the nanowire, $d_{m}$ is thus a non-negligible fraction of the wire lateral dimension, which explains the non-vanishing influence of edges on the apparent work function. This influence, however, is expected to decrease with the size of the sample, and vanish in the macroscopic limit. ${ }^{5}$ If we imagine that the nanowire crystal facets are scaled up in size, the symmetry of the resulting electrostatic potential will be retained. Larger spatial regions with the infinite-plane work function will develop along the facets, while the reduced-potential areas near the edges will remain essentially unchanged. 
Comparative $a b$ initio test calculations performed for a larger (7-atom thick) wire support this description. We also believe that analogous edge-induced reductions in potential should occur near crystal corners, where three equivalent $\mathrm{Al}(100)$ facets meet.

The electrostatic potential is not always reduced near facet edges. Fig. 4A shows the potential outside edges formed from $\mathrm{Al}(111)$ facets, without any ionic relaxation. At the acute edge between two $\mathrm{Al}(111)$ facets, the electrostatic potential is seen to be locally raised. This behavior can be understood by invoking the same type of mechanism - related to the open $p$-shell nature of $\mathrm{Al}$ - that is responsible for the anomaly in the $\mathrm{Al}$ work-function anisotropy, i.e., an anisotropic occupation of the atomic-like $p$-orbitals of the surface atoms. ${ }^{16}$ The latter mechanism yields for the $\mathrm{Al}(111)$ surface a work function which is lower than those of the $\mathrm{Al}$ (100) and (110) surfaces, at variance with Smoluchowski's rule. ${ }^{17}$ Pursuing our interpretation of work function anisotropy trends in aluminum, ${ }^{16}$ the local rise in potential outside the acute edge between the $\mathrm{Al}(111)$ low-work-function facets can be explained in terms of a change in the occupation numbers of the atomic-like $p$-orbitals on the edge atoms relative to the (111)-surface atoms. At a facet edge, the asymmetry between the three directional $p$-orbitals is different from that of an atom in an extended facet. Only one $p_{\|}$-orbital on the edge atom is parallel to both facets, whereas two $p_{\|}$-orbitals are parallel to an extended surface. Compared to atoms at the surface of extended $\mathrm{Al}(111)$ facets, a decrease in the occupation of the $p_{\|}$-density of states is induced at the edges. A corresponding increase in the occupation of the $p_{\perp}$-states perpendicular to the facet edges is created. At the acute edge, this redistribution of charge among the inequivalent orbitals of the edge atoms induces a local increase in potential ${ }^{16}$ outside the edge, which is stronger than the local potential reduction produced by the electronic edge-smoothing process.

This interpretation is supported by a comparative study with sodium facet edges. In Fig. 5, we present a $\mathrm{Na}$ nanowire with $60^{\circ}$ and $120^{\circ}$ edges between $\mathrm{Na}(110)$ facets. The work function of an infinite $\mathrm{Na}(110)$ surface was previously found to be $3.00 \mathrm{eV},{ }^{16}$ in good agreement with the electrostatic potential found here near the center of the $\mathrm{Na}$ facets. Compared to $\mathrm{Al}$, the general aspect of the potential outside the $\mathrm{Na}$ nanowire is considerably different. The potential shows extended regions of low work function at both the acute and the obtuse $\mathrm{Na}(110)$ edges. We believe this behavior represents the general case for metals (with the possible exception of transition metals ${ }^{31}$ ) which do not possess an open shell of $p$-states, and for edges between low-index equivalent facets.

The local work function around edges between two inequivalent facets is shown in Fig. 6A. The spatial behavior of the potential in vacuum is qualitatively different from that observed for equivalent facet edges. The potential rises continuously perpendicularly to the low-workfunction $\mathrm{Al}(111)$ facets, while it goes through a maximum perpendicularly to the high-workfunction $\mathrm{Al}(100)$ facets. At infinity, the apparent work function is intermediate between the two face-dependent work functions. Local edge effects clearly do not play a major role in determining the general behavior of the work function here, as work function anisotropy effects 
are stronger. The potential gradients that develop around edges, i.e., the macroscopic electric fields that exist outside the neutral metal crystal, indicate that this behavior is dominated by a different mechanism, namely charge transfer between facets. We will discuss this charge transfer mechanism in Sec. VI, where we present a model that explains our ab initio results and allows us to predict the dependence of the work function on the wire geometry in the macroscopic limit.

\section{EFFECT OF IONIC RELAXATION AT EDGES}

We have examined the influence of the outermost ionic layer relaxation on the electrostatic potential around our $\mathrm{Al}$ wires. The relaxed ionic positions for the three nanowires are displayed in Fig. 7. The corner ions are seen to relax strongly inward: at the edge between $\mathrm{Al}(100)$ facets, the corner ion is displaced by $4.1 \%$ of a lattice unit along a [110] direction, while at the acute (obtuse) edge between $\mathrm{Al}(111)$ facets, the corner ion is displaced by $2.7 \%$ (respectively $2.2 \%$ ) of a lattice unit. The nearest neighbors of the corner ion compensate this inward movement by relaxing slightly outward. At the acute angle between $\mathrm{Al}(100)$ and $\mathrm{Al}(111)$ facets, the corner ion moves by $10.3 \%$ of a lattice unit toward the metal interior. For comparison, the toplayer atoms at infinitely-extended $\mathrm{Al}(100)$ and $\mathrm{Al}(111)$ surfaces relax very little $(\sim 1 \%)$, in the outward direction. ${ }^{16}$ The trend of inward relaxation at facet edges is consistent with the electrostatic model proposed by Finnis and Heine ${ }^{32}$ based on Smoluchowski smoothing. The observed stronger relaxation of edge atoms is understood to result from an enhanced electronic smoothing around the facet edge.

The small surface relaxation at $\mathrm{Al}(100)$ facets does not significantly influence the electrostatic potential outside the metal. However, as shown in Figs. $4 \mathrm{~A}$ and $4 \mathrm{~B}$, the surface relaxation around edges between $\mathrm{Al}(111)$ facets has an important effect on the electrostatic potential outside the metal nanowire. When including the relaxation, the work function of a bulk $\mathrm{Al}(111)$ surface (4.25 $\mathrm{eV}$ ) is more closely approximated around the nanowire and a larger region of roughly constant potential develops. A similar situation is observed when comparing Figs. 6A and 6B, which respectively omit and include the surface ionic relaxation near an edge between inequivalent facets. Once again, the infinite-plane work functions are recovered better outside both the $\mathrm{Al}(100)$ and the $\mathrm{Al}(111)$ facets when the surface relaxation is included. We see that the ionic relaxation allows the infinite-plane work functions to be recovered closer to the edge. Whereas the ionic surface relaxation does not appreciably influence the work function of infinite metal surfaces, these results show that it has a non-negligible impact on the electrostatic potential near facet edges and around nanocrystals. 
VI. UNIFORM SURFACE DIPOLE MODEL FOR MACROSCOPIC CRYSTALS

The first-principles studies of nanowires presented above suggest that facet edges are characterized by a highly-localized ( $d_{m}$ of the order of a few angstroms) change in dipole density compared with infinite surfaces. In addition, in the case of inequivalent facet edges, the corresponding local edge effects play a relatively minor role in determining the general behavior of the electrostatic potential outside the wire. In macroscopic crystals, the localized change in dipole density near facet edges is a microscopic feature that can be neglected and the crystal facets can be viewed as carrying uniform densities of dipoles. In the following, we study the electrostatic potential and surface charges created by such a model system, as a method of understanding our $a b$ initio results for the inequivalent-facet wire and of predicting the influence of the size and shape of large crystals - inaccessible to ab initio computations - on the apparent work function. We note that in such macroscopic systems, as explained in Sec. IV, image contributions can be neglected and the local work function equated with the electrostatic potential.

We associate each crystal facet $i$ with a uniform dipole density corresponding to a given potential step $W_{i}$. In keeping with the results of our ab initio simulations, we focus essentially on metallic wires that preserve the bulk periodicity along the third dimension. For infinitelylong wires of arbitrary polygonal section, if we reference the electrostatic potential to zero at an infinite distance from the crystal, the potential $V^{d}$ induced by the planes of surface dipoles is analytically given at any point $\mathbf{x}$ in the $2 \mathrm{D}$ plane perpendicular to the wire by a weighted average over the crystal facets: ${ }^{33}$

$$
V^{d}(\mathbf{x})=\frac{1}{2 \pi} \sum_{i} s_{i} \Omega_{i}(\mathbf{x}) W_{i} .
$$

$\Omega_{i}(\mathbf{x})$ is the $2 \mathrm{D}$ angle subtending facet $i$, as seen from $\mathbf{x} . s_{i}$ is a sign coefficient equal to $-1(+1)$ if the surface dipole points away from (respectively towards) $\mathbf{x}$. This result can be generalized to three dimensions by considering solid angles $\Omega_{i}$ and normalizing the sum in Equ. 4 to $1 / 4 \pi{ }^{33}$

If all the crystal facets possess the same potential step $W_{0}$, Equ. 4 shows that the electrostatic potential outside the metal is constant and equal to zero. Inside the crystal, the dipole potential $V^{d}(\mathbf{x})$ is also uniform, as required in a metallic system (viewed on a macroscopic scale), and is equal to $V^{d}(\mathbf{x})=-W_{0}$. We can identify $W_{0}$ with the work function of the facets, since the Fermi energy only contributes a fixed potential shift inside the metal. From Equ. 4 we thus recover the intuitive result that the apparent work function $\bar{W}$ of a finite macroscopic crystal with equivalent facets is given by the work function $W_{0}$ obtained for an infinite surface. This result is independent of the angles between the facets and is valid for crystals of macroscopic dimensions. For sufficiently large samples showing equivalent facets, the apparent work function is thus independent of its global morphology. If the facets are reduced to a few atomic planes however, and in the standard case of a reduction in dipole density at the facet edge, the apparent work function $\bar{W}$ will be slightly lower than the work function $W_{0}$ of an infinitely-extended facet. 
If a finite metallic crystal is bounded by facets with different dipole distributions (different potential steps $\left.W_{i}\right)$, the surface dipoles create a potential $V^{d}(\mathbf{x})$ which is not constant inside the metal. Surface charges have therefore to develop spontaneously on the crystal facets to allow a macroscopically-constant potential in the metal to coexist with the face-dependent surface dipoles. We include thus a second ingredient in the model, i.e., a surface charge distribution $\sigma(\mathbf{x})$. This surface charge density induces a supplementary electrostatic potential $V^{\sigma}(\mathbf{x})$ that compensates the variation of the dipole potential $V^{d}(\mathbf{x})$ inside the metal, and recovers the physical requirement of a constant potential in the metal interior. Therefore, we impose $V^{\sigma}(\mathbf{x})$ equal, inside the metal, to the opposite of the dipole potential, within a constant $W$ :

$$
V^{\sigma}(\mathbf{x})=-V^{d}(\mathbf{x})-W, \text { for } \mathbf{x} \text { inside the metal. }
$$

In this way, the sum of the potentials induced by the dipoles and the charges is constant inside the crystal. The value of the constant $W$ is fixed by the requirement that the total surface charge vanishes, since the crystal is nominally uncharged and globally neutral. If $S$ is the crystal surface, we impose:

$$
\int_{S} \sigma(\mathbf{x}) d s=0
$$

We determine the surface charge numerically using an iterative technique similar to the charge simulation method. ${ }^{34-36}$ We first produce a potential $-V^{d}(\mathbf{x})-W$ inside the metal, with an assumed constant $W$, by arranging a dense set of discrete charges (or lines of charge in the case of wires) on the surface $S$. The total charge necessary is examined, the constant $W$ is adjusted to reduce it, and the process is repeated. When the total charge vanishes, the surface charge density has been determined and the corresponding potential constant $W$ gives the apparent work function $\bar{W}$. The surface charge density depends on the entire crystal geometry and produce a potential $V^{\sigma}$ which adds to the dipole potential variation in the vacuum. In general, $\bar{W}$ is an intermediate value between all the face-dependent work functions $W_{i}$.

Geometrical considerations of Equ. 4 show that the electrostatic potential follows a number of scaling laws that we can conveniently exploit. We note that $V^{d}(\mathbf{x})$ depends only on a set of angles $\Omega_{i}(\mathbf{x})$. If the crystal dimensions, collectively written as $\left\{L_{i}\right\}$, are homogeneously expanded by a factor $\lambda$, keeping all angles fixed, we note that the potential is globally unchanged, in the sense that:

$$
V_{\left\{\lambda L_{i}\right\}}^{d}(\lambda \mathbf{x})=V_{\left\{L_{i}\right\}}^{d}(\mathbf{x})
$$

The potential $V_{\left\{L_{i}\right\}}^{\sigma}(\mathbf{x})$ must follow the same scaling law, which leads to a surface charge density that is inversely proportional to the size of the crystal (in two or three dimensions):

$$
\sigma_{\left\{\lambda L_{i}\right\}}(\lambda \mathbf{x})=\frac{1}{\lambda} \sigma_{\left\{L_{i}\right\}}(\mathbf{x}) .
$$

This observation is independent of the crystal geometry and leads to minute charge densities in macroscopic crystals. We have thus demonstrated that the apparent work function $\bar{W}$ does not depend on the size of the crystal but only on its global shape. 
We now consider the special case of metallic wires that have a parallelogram section with facets of lengths $A$ and $B$, and work functions $W_{A}$ and $W_{B}$ respectively (see Fig. 8). From Equ. 4, we see that the electrostatic potential, outside the crystal, is a linear function of the difference in the two work functions only. It is convenient, in the following discussion, to take the potential inside the metal as the reference energy for $V(\mathbf{x})=V^{d}(\mathbf{x})+V^{\sigma}(\mathbf{x})$ and to decompose the electrostatic potential $V(\mathbf{x})$ into:

$$
V(\mathbf{x})=\left\{\begin{array}{lr}
0 & \text { if } \mathbf{x} \text { is inside the metal } \\
\left(W_{A}-W_{B}\right) v_{A, B, \alpha}(\mathbf{x})+W_{B} \text { otherwise }
\end{array}\right.
$$

$v_{A, B, \alpha}(\mathbf{x})$ is a reduced potential that depends on the facet lengths $A$ and $B$ as well as on the angle between the facets $\alpha$, but is independent of the work functions $W_{A}$ and $W_{B}$. Using this definition, the reduced potential $v_{A, B, \alpha}(\mathbf{x})$ varies from 0 (just outside facet $B$ ) to 1 (outside facet $A)$ in the vacuum. The potential scaling is now: $v_{\lambda A, \lambda B, \alpha}(\lambda \mathbf{x})=v_{A, B, \alpha}(\mathbf{x})$, so that we only need consider reduced potentials $v_{m, \alpha}(\mathbf{x})$ that depend on the angle $\alpha$ and the aspect ratio $m=A / B$. The apparent work function $\bar{W}$ is written (using Equ. 9):

$$
\bar{W}=\left(W_{A}-W_{B}\right) \bar{w}_{m, \alpha}+W_{B},
$$

where $\bar{w}_{m, \alpha}$ is the value of $v_{m, \alpha}(\mathbf{x})$ at an infinite distance from the crystal.

In Fig. 8 we show a contour plot of the total reduced potential $v_{m, \alpha}(\mathbf{x})=v^{d}(\mathbf{x})+v^{\sigma}(\mathbf{x})$ outside a crystal with an aspect ratio $m=A / B=1$. The angle between the facets is equal to that between a (111) and a (100) fcc surface. We observed that the model reproduces well the general behavior of the $a b$ initio potential outside the $\mathrm{Al}$ wire with inequivalent facets in Fig. 6 . For wires with facets of equal length the apparent work function is equal to the average of the face-dependent values: $\bar{W}=\left(W_{A}-W_{B}\right) / 2$ (i.e., $\left.\bar{w}=0.5\right)$, independently of the angle $\alpha$ between the facets. The surface charge density obtained from the model is shown in the lower panel of Fig. 8 and is seen to be highly inhomogeneous. For the wires studied here, the facets of higher work function carry a negative charge. In view of the two different work functions coexisting at each facet edge, the potential is discontinuous along the outside perimeter of the parallelogram. This leads to a surface charge density that diverges at each vertex of the parallelogram. ${ }^{37,38}$ However, at real facet edges, the smooth variation of surface dipole density near the facet edge is expected to induce a finite charge transfer between the various crystal facets.

In Fig. 9 we show a contour plot of the total reduced potential $v_{m, \alpha}(\mathbf{x})=v^{d}(\mathbf{x})+v^{\sigma}(\mathbf{x})$ outside a crystal with a facet ratio $m=A / B=1 / 3$. The angle between the facets is again equal to that between a (111) and a (100) surface. The surface charge density is shown in the lower panel of Fig. 9. We note that it is lower on the larger facet, and the apparent work function $(\bar{w}=0.344)$ is biased towards the larger facet.

With the model one can thus predict in general the dependence of the local work function and also of the apparent work function on the wire geometry. In Ref. 5, we have examined in detail the dependence of the apparent work function $\bar{w}_{m, \alpha}$ on the wire aspect ratio $m$ and 
angle $\alpha$. The model predictions show that the aspect ratio is the crucial factor determining the apparent work function, while the angle between the facets has only a limited influence. In addition, the predicted dependence of the apparent work function on the aspect ratio is found to differ significantly from the result of the commonly used surface-weighted average rule, which is expected thus to apply only to selected cases of simple surface geometries (i.e., infinite planes, cylinders, and spheres). ${ }^{39}$

\section{CONCLUSIONS}

In this study, we have performed $a b$ initio calculations for metal nanowires with facets of different crystallographic orientations to investigated the work-function profile near facet edges. We have then modeled the $a b$ initio surface charge distribution to derive the dependence of the work function on the crystal geometry in the macroscopic limit. Our ab initio results for the electrostatic potential around sharp equivalent facet edges show a much more complex behavior than in previous theoretical studies. Comparing aluminum and sodium facet edges, we find a variety of different behaviors of the local work function near the edges, which can be understood by considering the particular filling of atomic-like orbitals of the edge atoms. The relaxation of the surface ions near facet edges influences the electrostatic potential in the vacuum more strongly than in the case of infinite planar surfaces. Our results on inequivalent-facet edges show for the first time how different work functions can coexist on either side of a facet edge, provided a non-vanishing surface charge distribution develops on the crystal faces. The surface charge is most intense near sharp edges and contributes to creating a macroscopically-flat electrostatic potential inside the metal. By extrapolating from our ab initio results for nanocrystals, we can predict the work-function behavior around larger, macroscopic crystals with non-equivalent facets, and derive the dependence of the apparent work function on the crystal geometry.

\section{Acknowledgements}

One of us (CJF) acknowledges support from the Swiss National Science Foundation under grant No. 20-55811.98. The calculations were performed at EPF-Lausanne and the CSCS in Manno, Switzerland. 


\section{Appendix}

In order to describe the local work function outside a metallic surface, the exchangecorrelation contribution must be included in the total potential felt by an electron. The LDA fails to reproduce the long-range image potential, which the correct exchange-correlation potential is known to follow. Classical electrostatics shows that outside an infinite metal surface the image potential felt by an electron is given by $-e^{2} / 4 r$, where $r$ is the distance to the metal surface, and $e$ the electron charge (atomic units are assumed here). While the classical image potential diverges at the metal surface, it describes correctly the exchange-correlation potential felt by an electron in the vacuum beyond the range of the surface electronic density, provided that $r$ is taken as the distance to the effective image-plane surface. ${ }^{40}$ On the contrary, the LDA potential decays exponentially in the vacuum with the charge density, and is therefore qualitatively incorrect far from the surface.

For a classical point charge in the vicinity of a conducting wedge, the electrostatic potential scattered by the wedge was recently established analytically in terms of a collection of image sources. ${ }^{41}$ The locations and values of the virtual images depend on the position of the source charge and the angle of the wedge. Using the formalism developed in Ref. 41, we have calculated the image potential felt by a classical electron outside a metallic facet edge, by integrating the work performed against the image force on taking the source electron to infinity. In Fig. 10 we have plotted the classical image potential around both a $90^{\circ}$ and an acute-angle wedge, corresponding to a (100)-(010) facet edge and a (100)-(111) facet edge, respectively. In both cases, the image potential behaves monotonously around the facet edge, recovering within a few tens of atomic units a form characteristic of an infinite crystal surface. These results show that the image contribution near a facet edge is smoothly behaved and is negligible beyond $\sim 100 \mathrm{~nm}$. The local work function variation from facet to facet is thus accounted for by the electrostatic contribution only. 


\section{References}

1 J. Hölzl and F. K. Schulte, in Solid Surface Physics, edited by G. Höhler and E. A. Niekisch (Springer-Verlag, Berlin, 1979), vol. 85 of Springer Tracts in Modern Physics, chap. 1, pp. $1-150$.

2 Y. Yamamoto and T. Miyokawa, J. Vac. Sci. Technol. B 16, 2871 (1998).

3 C. H. Hinrichs, W. A. Mackie, I. Cohen, J. Alin, D. Schnitzler, and I. Noel, Rev. Sci. Instrum. 65, 3689 (1994).

4 R. M. Wolf, J. W. Bakker, and B. E. Nieuwenhuys, Surf. Sci. 246, 420 (1991).

5 C. Fall, N. Binggeli, and A. Baldereschi, Phys. Rev. Lett. (in press).

6 L. L. Kesmodel and L. M. Falicov, Solid State Comm. 16, 1201 (1975).

7 G. Schreckenbach, R. Kaschner, and P. Ziesche, Phys. Rev. B 46, 7864 (1992).

8 M. C. Desjonquères and D. Spanjaard, Concepts in Surface Physics (Springer-Verlag, Berlin, 1996), 2nd ed.

9 V. T. Binh, Surf. Sci. 202, L539 (1988).

10 N. D. Lang and W. Kohn, Phys. Rev. B 3, 1215 (1971).

11 R. Monnier, J. P. Perdew, D. C. Langreth, and J. W. Wilkins, Phys. Rev. B 18, 656 (1978).

12 V. Sahni, J. P. Perdew, and J. Gruenebaum, Phys. Rev. B 23, 6512 (1981).

13 M. Heinrichsmeier, A. Fleszar, and A. G. Eguiluz, Surf. Sci. 285, 129 (1993).

14 L. Reinaudi, M. Del Popolo, and E. Leiva, Surf. Sci. 372, L309 (1997).

15 J. K. Grepstad, P. O. Gartland, and B. J. Slagsvold, Surf. Sci. 57, 348 (1976).

16 C. J. Fall, N. Binggeli, and A. Baldereschi, Phys. Rev. B 58, R7544 (1998).

17 R. Smoluchowski, Phys. Rev. 60, 661 (1941).

18 D. M. Ceperley and B. J. Alder, Phys. Rev. Lett. 45, 566 (1980).

19 N. Troullier and J. L. Martins, Phys. Rev. B 43, 1993 (1991).

20 L. Kleinman and D. M. Bylander, Phys. Rev. Lett. 48, 1425 (1982).

21 H. J. Monkhorst and J. D. Pack, Phys. Rev. B 13, 5188 (1976).

22 C.-L. Fu and K.-M. Ho, Phys. Rev. B 28, 5480 (1983).

23 The parameters used for $\mathrm{Al}(111)$ are an $9+6$ supercell (9 layers of $\mathrm{Al}$, and 6 equivalent layers of vacuum) with 36 reduced k-points. The corresponding values for $\mathrm{Al}(100)$ and $\mathrm{Al}(110)$ are 
respectively a $8+6$ cell with $45 \mathrm{k}$-points and a $8+8$ cell with 48 k-points.

24 J. P. Perdew, K. Burke, and M. Ernzerhof, Phys. Rev. Lett. 77, 3865 (1996).

25 M. Heinrichsmeier, A. Fleszar, W. Hanke, and A. G. Eguiluz, Phys. Rev. B 57, 14974 (1998);

I. D. White, R. W. Godby, M. M. Rieger, and R. J. Needs, Phys. Rev. Lett. 80, 4265 (1998).

26 C. R. Anderson, SIAM J. Sci. Stat. Comput. 13, 923 (1992).

27 For the bulk calculation of the Fermi energy, we use a cut-off of 36 Ry and 570 reduced k-points.

28 C. J. Fall, N. Binggeli, and A. Baldereschi, J. Phys. C: Condens. Matter 11, 2689 (1999).

29 H. Ishida and A. Liebsch, Phys. Rev. B 46, 7153 (1992).

30 Y. Hasegawa, J. F. Jia, K. Inoue, A. Sakai, and T. Sakurai, Surf. Sci. 386, 328 (1997).

31 C. J. Fall, N. Binggeli, and A. Baldereschi, Phys. Rev. B 61, 8489 (2000).

32 M. W. Finnis and V. Heine, J. Phys. F: Metal Phys. 4, L37 (1974).

33 E. Durand, Électrostatique, vol. I-II (Masson \& Cie, Paris, 1966).

34 H. Singer, H. Steinbigler, and P. Weiss, IEEE Trans. Power Apparatus and Systems PAS-93, 1660 (1974).

35 N. H. Malik, IEEE Trans. Elec. Insulation 24, 3 (1989).

36 Contrary to some applications of the charge simulation method, the surface charge densities calculated here are real.

37 N. A. Burnham, R. J. Colton, and H. M. Pollock, Phys. Rev. Lett. 69, 144 (1992).

38 J. E. Inglesfield, Phys. Rev. Lett. 70, 246 (1993).

39 C. Herring and M. H. Nichols, Rev. Mod. Phys. 21, 185 (1949).

40 N. D. Lang and W. Kohn, Phys. Rev. B 7, 3541 (1973).

41 K. I. Nikoskinen and I. V. Lindell, IEEE Trans. on Antennas and Propagation 45, 179 (1995). 


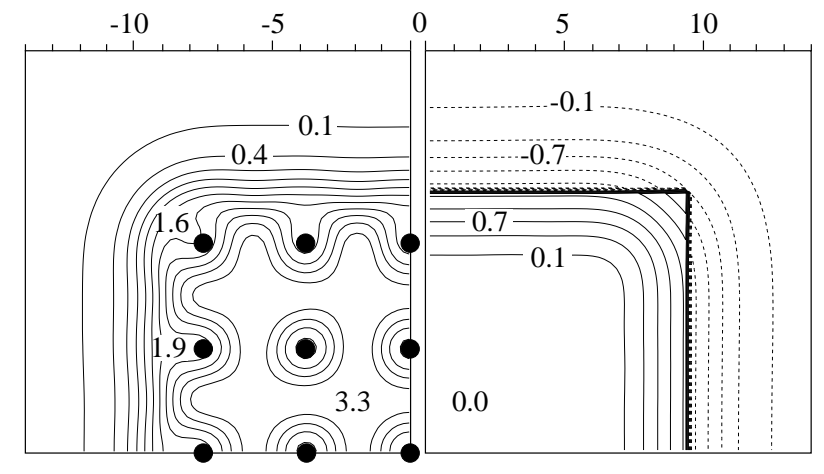

FIG. 1: Two-dimensional average valence electron density (left panel) and total macroscopic charge density (right panel) at a facet edge between two $\mathrm{Al}(100)$ surfaces with no surface relaxation. Dashed lines correspond to negative values. The contour lines are uniformly spaced by 0.3 electrons per unit cell. The black disks indicate the atomic columns. After macroscopic averaging, the ionic charge is inside rectangular region enclosed by the thick solid line in the right panel. 


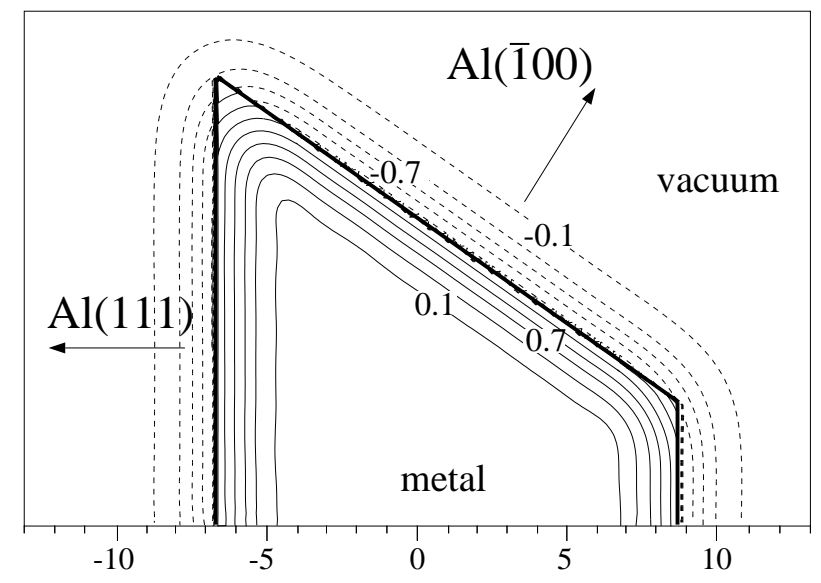

FIG. 2: Contour plot of the total macroscopic charge density at the two inequivalent facet edges between $\mathrm{Al}(100)$ and $\mathrm{Al}(111)$ surfaces, without surface relaxation. The contour lines are uniformly spaced by 0.3 electrons per unit cell. Dashed lines correspond to negative values. The thick solid line encloses the macroscopic ionic charge density.

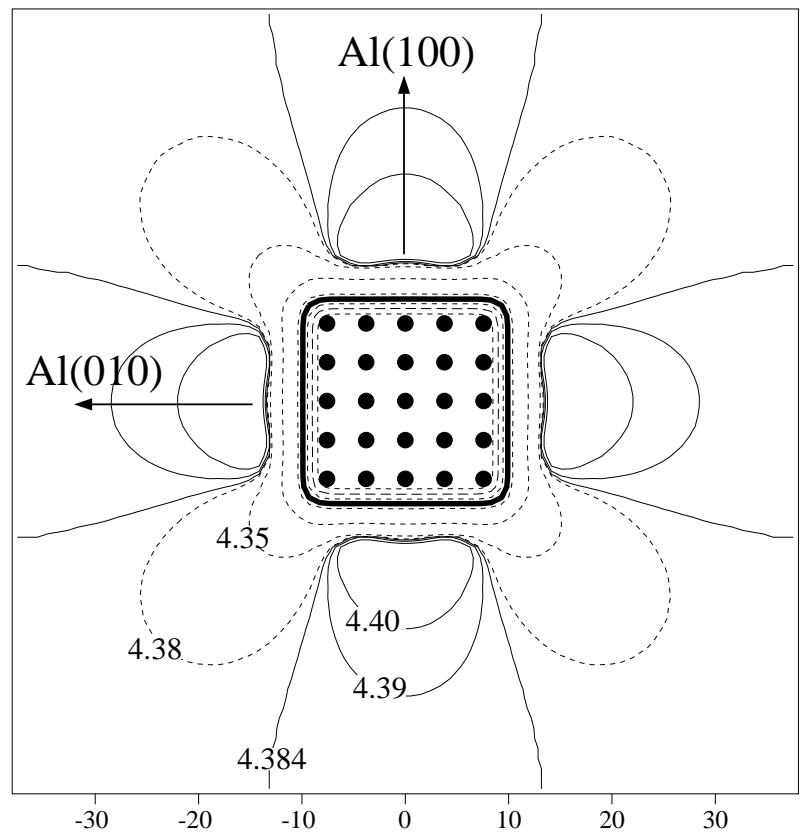

FIG. 3: Contour plot of the macroscopic electrostatic potential (in $\mathrm{eV}$ ) around an $\mathrm{Al}$ nanowire bounded by (100) facets, without surface relaxation. The potential zero (thick line) has been set at the Fermi energy. Continuous (dashed) lines indicate regions above (respectively below) the potential at infinity. The black disks indicate the atomic columns. The axes are graduated in atomic units. 

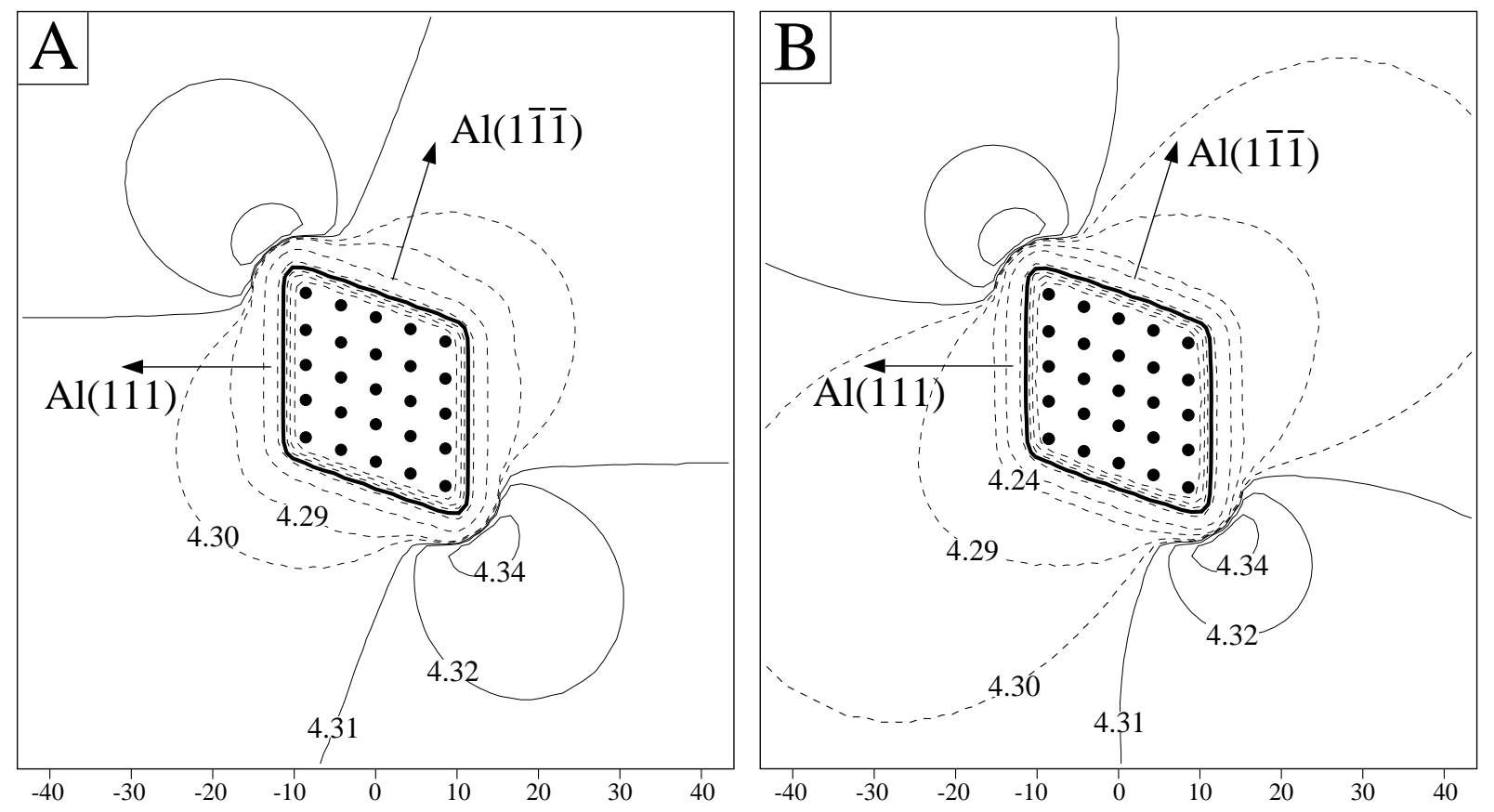

FIG. 4: Contour plot of the macroscopic electrostatic potential (in eV) around a nanowire containing facet edges between $\mathrm{Al}(111)$ surfaces. The left (right) panel omits (respectively includes) surface relaxation. The potential zero (thick line) is set at the Fermi energy. Continuous (dashed) lines indicate regions above (respectively below) the potential at infinity. The black and white disks indicate the atomic columns. The axes are graduated in atomic units. 


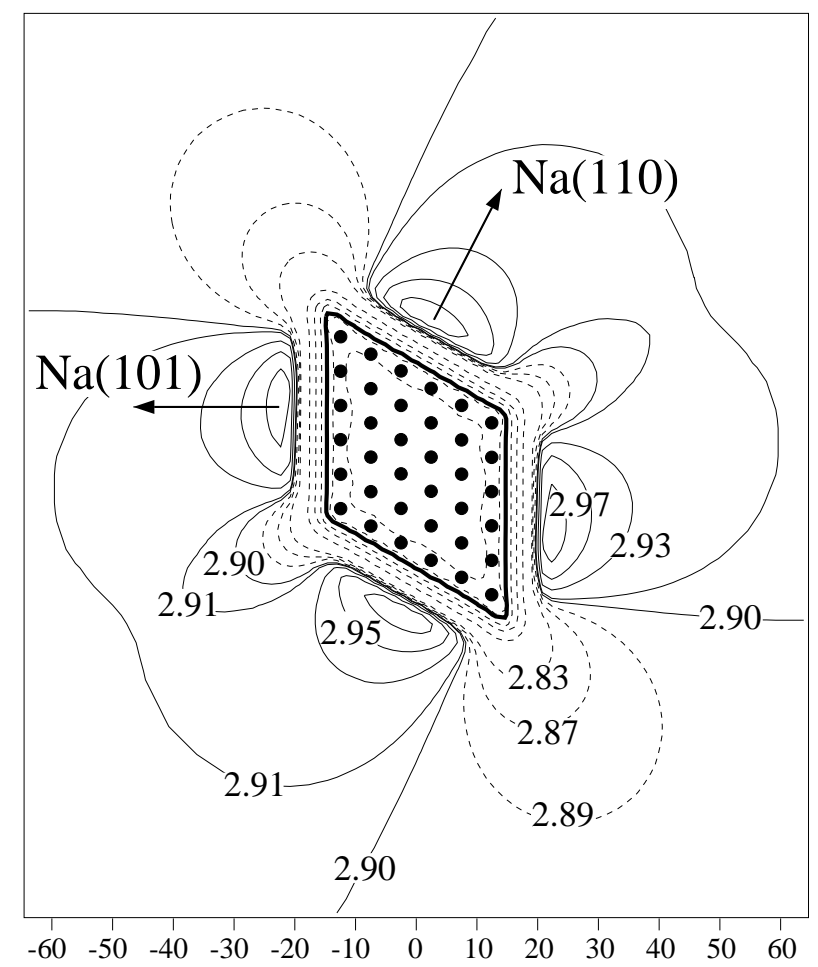

FIG. 5: Contour plot of the macroscopic electrostatic potential (in $\mathrm{eV}$ ) around an unrelaxed nanowire describing $60^{\circ}$ and $120^{\circ}$ edges between $\mathrm{Na}(110)$ facets. The potential zero (thick line) is set at the Fermi energy. Continuous (dashed) lines indicate regions above (respectively below) the potential at infinity. The disks indicate the atomic columns. The axes are graduated in atomic units. 

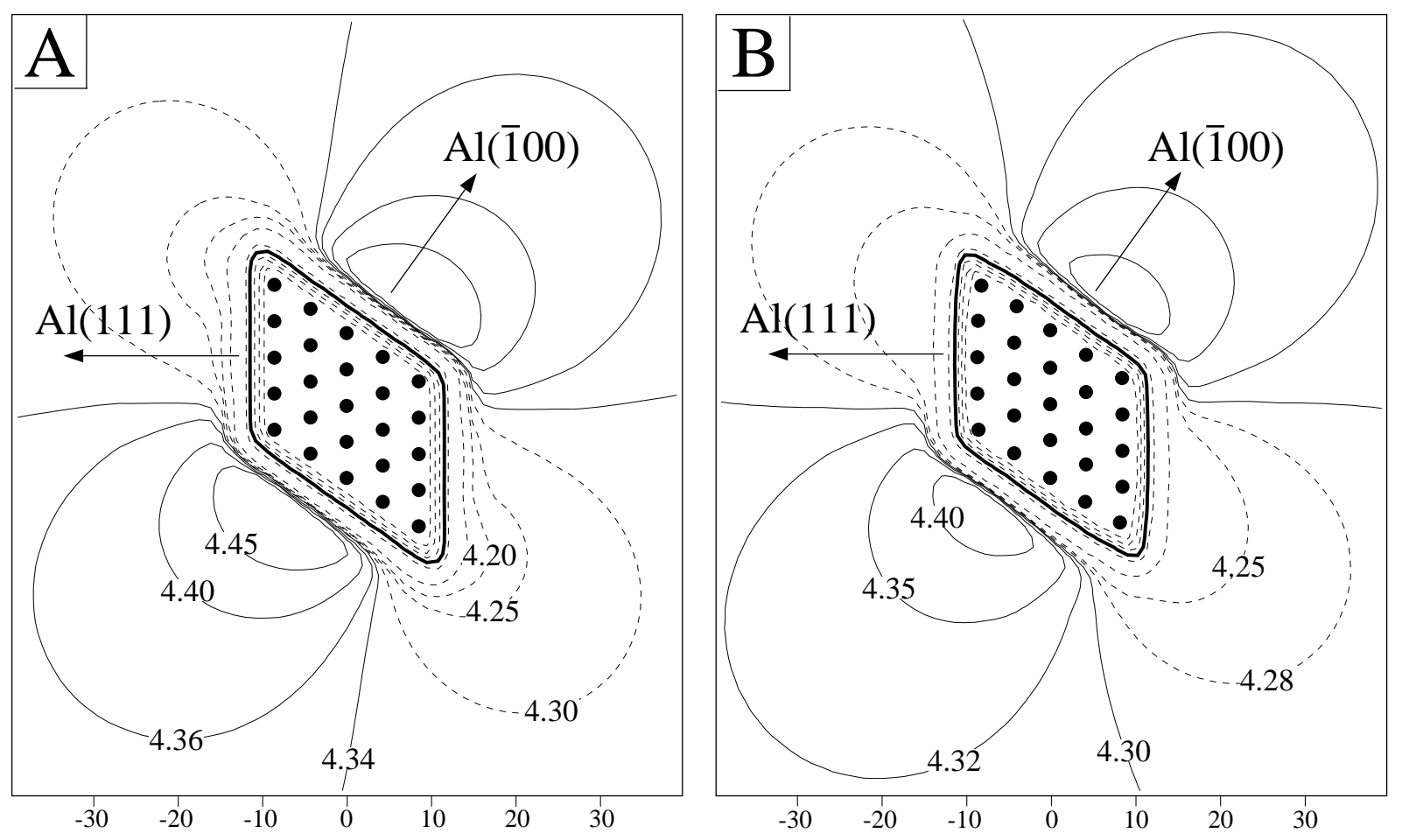

FIG. 6: Same as Fig. 4 but for edges between $\mathrm{Al}(100)$ and $\mathrm{Al}(111)$ facets. 


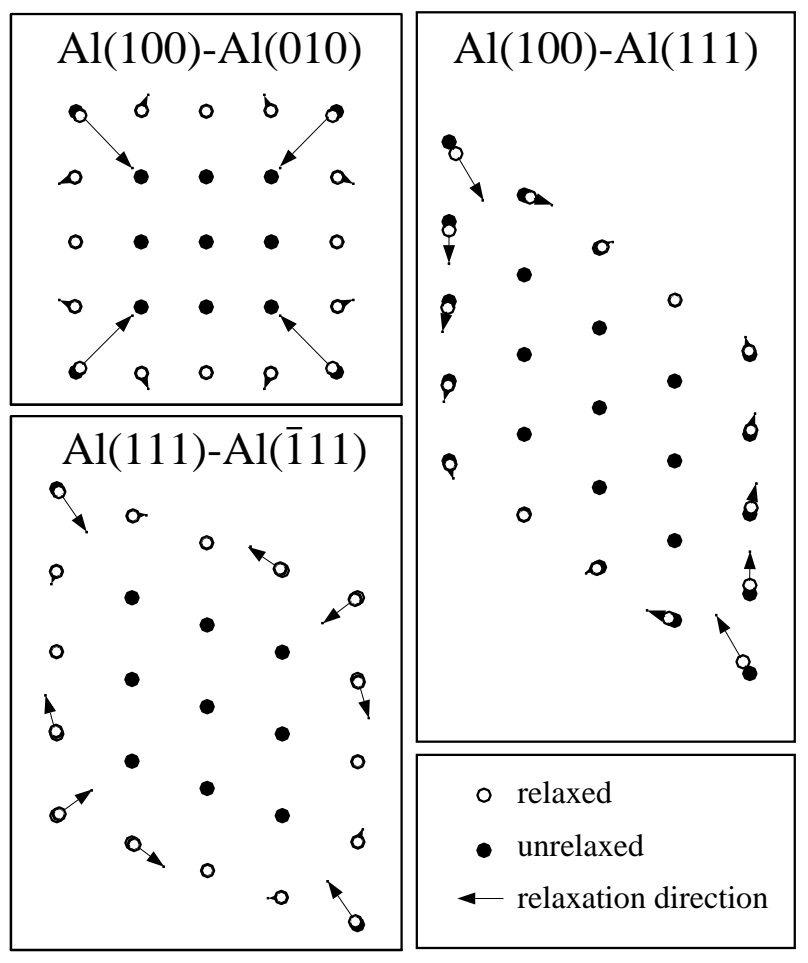

FIG. 7: Relaxation of surface atoms near edges between $\mathrm{Al}(100)$ and $\mathrm{Al}(111)$ facets. The black circles indicate the unrelaxed atomic columns, and the white circles the relaxed positions. The arrows indicate the direction of the relaxation, and are proportional to the relaxation distance, multiplied by a factor 15 for the $\mathrm{Al}(100)-\mathrm{Al}(010)$ and $\mathrm{Al}(111)-\mathrm{Al}(11 \overline{1})$ edges (left panels) and by a factor 5 for the $\mathrm{Al}(100)-\mathrm{Al}(111)$ edges (right panel). 


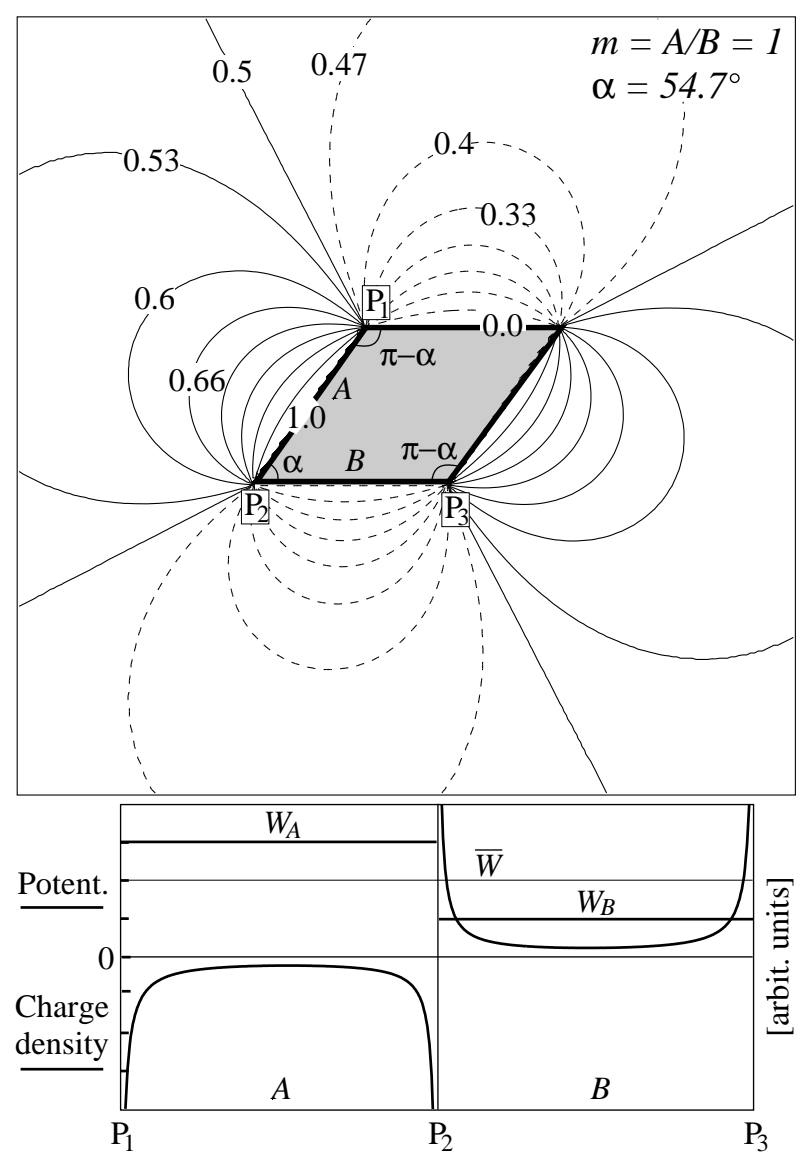

FIG. 8: Upper panel: Reduced electrostatic potential $v_{m, \alpha}(\mathbf{x})$ (defined in the text) outside a model crystal with non-equivalent facets of length $A$ and $B$. The aspect ratio is chosen such that $A=B$. The angle between the facets is $\alpha=54.7^{\circ}$. Continuous (dashed) indicate regions above (respectively below) the potential at infinity. Lower panel: Potential just outside the crystal surface (dashed lines) — equal to $W_{A}$ and $W_{B}$ for facets $A$ and $B$ respectively — and surface charge density on each facet (solid line). The reduced apparent work function is $\bar{\omega}=0.5$. 


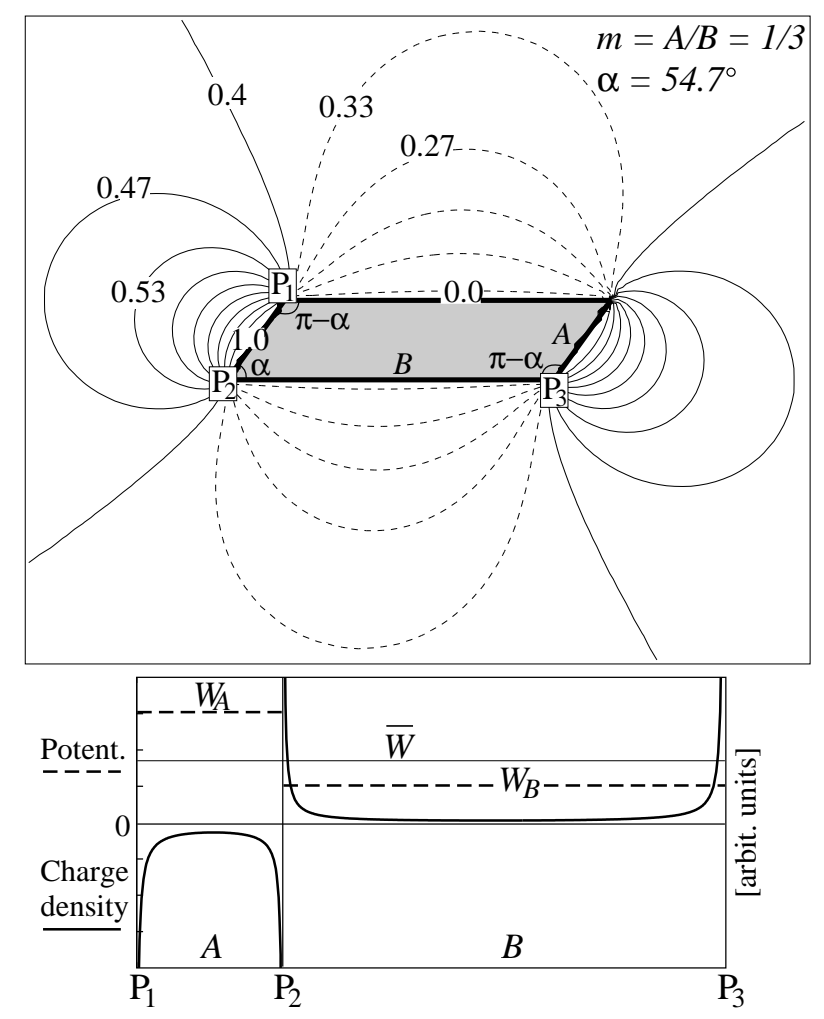

FIG. 9: Same as Fig. 8, but for an aspect ratio $A / B=1 / 3$. The reduced apparent work function is $\bar{\omega}=0.344$.

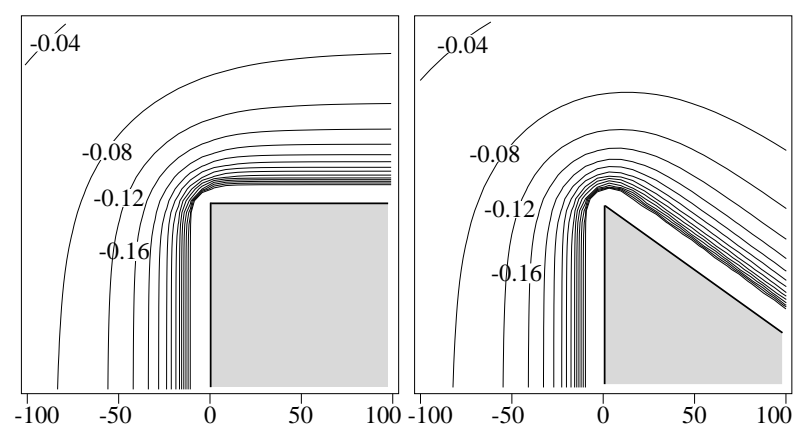

FIG. 10: Classical image potential (in eV) near a metallic wedge. Left panel: $90^{\circ}$ wedge. Right panel: $54.7^{\circ}$ wedge, corresponding to an edge between a (111) and a (100) surface. The contour lines are equally spaced. The axes are graduated in atomic units. 